\title{
A higher form (of) mediation
}

\author{
Herman Verlinde, ${ }^{a}$ Lian-Tao Wang, ${ }^{a}$ Martijn Wijnholt ${ }^{b}$ and Itay Yavin ${ }^{a}$ \\ ${ }^{a}$ Physics Department, Princeton University, Joseph Henry Laboratories, \\ Princeton NJ 08540, U.S.A. \\ ${ }^{b}$ Max-Planck-Institut für Gravitationsphysik, Albert-Einstein-Institut, \\ Potsdam, Germany \\ E-mail: verlinde@princeton.edu, lianwang@princeton.edu, wijnholt@aei.mpg.de, \\ iyavin@princeton.edu
}

ABSTRACT: We exhibit a simple and robust mechanism for bulk mediation of supersymmetry breaking between hidden and visible sectors localized on geometrically separated D-branes in type II string theory. The mediation proceeds via $\mathrm{RR}$ p-forms that couple via linear Chern-Simons terms to the abelian vector bosons on the branes. From a 4 -d low energy perspective, the mechanism reduces to $\mathrm{U}(1)$ mediation.

KEYwords: D-branes, Supersymmetry Breaking, Supersymmetry Phenomenology. 


\section{Contents}

1. Introduction 且

1.1 U(1) mediated supersymmetry breaking 3

2. RR-form mediation 5

2.1 Phenomenology with branes

$2.2 \mathrm{U}(1)$ mediation via RR-forms

2.3 Low energy parameters 11

3. Summary and discussion 12

A. U(1) mediation in the heterotic string 13

\section{Introduction}

A key component in string phenomenology is to find a good mechanism of supersymmetry breaking, and for mediating its effect to the standard model. In typical phenomenological scenarios, SUSY breaking takes place in a hidden sector of particles without direct couplings to the standard model particles. The two sectors communicate via messenger particles, that mediate the SUSY breaking effects and thereby induce a distinctive pattern of MSSM soft terms. While there are several promising phenomenological scenarios of mediated SUSY breaking, there has been only limited success in finding robust realizations in string theory.

A logical setting for scenarios with messenger mediated supersymmetry breaking is via string models in which the standard model is localized on branes [1 - 3 . The decoupling of the hidden and visible sector is then achieved via geometric separation in the extra dimensions. A typical set-up is indicated in figure 1. It consists of a visible brane configuration localized in some region $\varnothing$, that we assume realizes the MSSM, or some viable extension thereof. We assume that supersymmetry is broken on the hidden brane located at $\diamond$. In this paper we will exhibit, in this general set-up, a simple and robust new mechanism for mediating SUSY breaking to the MSSM sector.

In the set-up of figure 1, the hidden brane communicates with the MSSM brane via closed string fields - graviton, dilaton and p-form fields - that traverse the Calabi-Yau geometry. Which particular bulk field dominates the mediation depends on specific details, such as moduli stabilization, presence of warping, and other properties of the compactification geometry [5-8]. This sensitivity on details of the Planck scale geometry is a precarious aspect of all bulk mediation mechanisms, that typically makes it hard to extract robust, universal predictions. A concrete danger is that the soft terms may induce new $\mathrm{CP}$ or flavor violations, that would conflict with current experimental bounds. An attractive Planck 


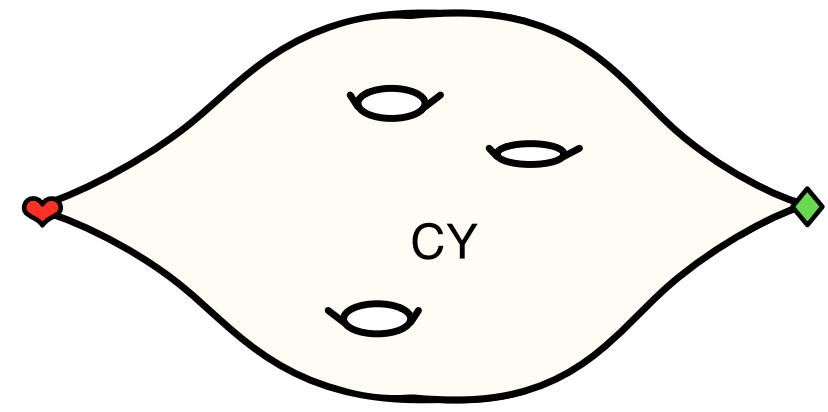

Figure 1: In our set-up, the MSSM and the SUSY breaking hidden sector each live on collections of branes localized within separate small regions of the Calabi-Yau manifold.

scale scenario, that manages to avoid these problems, is anomaly mediation [9]. It relies on the assumption that all bulk modes except gravity are massive compared to the distance between the hidden and visible brane. The mediation then proceeds via the superconformal anomaly. In its minimal form, however, it predicts a negative mass squared for the sleptons. Recent studies that investigate string realizations of anomaly mediation include [5, 6, 8].

Another promising mechanism for communicating SUSY breaking is gauge mediation [10]. In this class of models, the SUSY breaking scale can be much lower. The messenger fields are chiral multiplets, that couple to the hidden sector and carry standard model gauge charges. Flavor violations are suppressed, because the messengers couple to the visible sfermions via universal gauge interactions. In brane realizations, gauge mediation proceeds via open strings stretched between the visible and hidden sector branes. The branes must therefore be placed at a small sub-stringy relative distance [7].

Based on the available scenarios, one may conclude that there are two basic possibilities:

1. The separation $d$ between the branes is larger than the string length

$$
d \gg \ell_{s}
$$

and the supersymmetry breaking takes place via Planck scale mediation, effectuated by closed string exchange. Soft masses are of order $\langle F\rangle / M_{\mathrm{Pl}}$ or smaller and the SUSY breaking scale must be higher than the geometric mean of the electro-weak and Planck scale.

2. The hidden and visible branes are at sub-stringy relative distance

$$
d \ll \ell_{s}
$$

SUSY breaking then takes place via gauge mediation, brought about by light open strings of mass $M=d / \ell_{s}^{2}$. The SUSY breaking scale can now be much smaller than the Planck scale. The soft masses are of order $\langle F\rangle / M$ with $M$ the messenger scale. 
In this paper we will uncover a third possibility: we will exhibit a simple mechanism in which both the closed and open string modes participate in the communication of supersymmetry breaking. Moreover, the mechanism works irrespective of the brane separation, and both low and high scale supersymmetry breaking are allowed. This new mediation scenario manifestly respects flavor symmetry and relies on the coupling between $U(1)$ vector bosons on the branes and the RR-forms in the bulk. In a suitable set-up, it can dominate over all other mediation mechanisms.

\section{$1.1 \mathrm{U}(1)$ mediated supersymmetry breaking}

Phenomenological string models frequently lead to standard model-like gauge theories with additional $\mathrm{U}(1)$ factors. In case both the visible and hidden sector particles are charged under a common $\mathrm{U}(1)$ gauge group, the associated $Z^{\prime}$-boson can be responsible for the mediation of supersymmetry breaking. The phenomenology of such U(1) mediation scenarios, in which the supersymmetry breaking is parametrized by the gaugino mass, was recently considered in [1]. The low energy action takes the schematic form

$$
\mathcal{L}=\mathcal{L}_{\mathrm{MSSM}}\left(Q, \mathcal{A}^{\prime}\right)+\mathcal{L}_{\text {HIDDEN }}\left(X, \mathcal{A}^{\prime}\right)
$$

Here $Q$ and $X$ denote the MSSM and hidden sector fields and $\mathcal{A}^{\prime}$ the $\mathrm{U}(1)$ gauge field. In the hidden sector, supersymmetry is broken by some F-term $\operatorname{VEV~}\langle F\rangle$. On the visible brane, SUSY breaking is driven by the mass splitting between the $\mathcal{A}^{\prime}$ boson and its superpartner $\lambda^{\prime}$.

In its minimal form, $\mathrm{U}(1)$ mediation realizes an intermediate version of split supersymmetry. All visible matter multiplets that are charged under the $\mathrm{U}(1)$ receive soft mass terms at 1-loop, while the gauginos, which do not directly couple to $\mathcal{A}^{\prime}$, acquire a mass at the 2-loop level. The observed lower bound on the gaugino mass (of about $100 \mathrm{GeV}$ ) thus forces the sfermion mass to be of order $100 \mathrm{TeV}$. To generate the electroweak scale, one must accept a finetuning of order $10^{-6}$. A more detailed discussion of the phenomenology of $\mathrm{U}(1)$ mediation is found in [11].

In this note, we point out how $\mathrm{U}(1)$ mediation is naturally realized in string theory. Having such a embedding at hand is of particular interest if it allows for direct investigation of the required tuning mechanism via the multitude of vacua in the string landscape. For this reason, we will imagine a set-up as in figure 1, with the hidden and visible sector on branes located at local regions of the Calabi-Yau manifold, each at the bottom of a flux stabilized warped throat [12, 13]. The discrete tuning of the flux then provides explicit control over the relative ratio between the string scale and the low energy scale on the brane.

At first sight, the set-up of figure 1 looks like the wrong starting point for U(1) mediation: since both branes are separated by a large distance compared the string scale, they do not share any low energy open string modes. The two branes each have their own worldvolume gauge theory, with each their own $\mathrm{U}(1)$ vector boson, $\mathcal{A}_{V}$ and $\mathcal{A}_{H}$. The open string action therefore splits up as (here $Q$ and $X$ encode all other visible and hidden sector fields)

$$
\mathcal{L}_{\odot}+\mathcal{L}_{\diamond}=\mathcal{L}_{\mathrm{MSSM}}\left(Q, \mathcal{A}_{V}\right)+\mathcal{L}_{\mathrm{HIDDEN}}\left(X, \mathcal{A}_{H}\right)
$$


This is clearly not a suitable action for any mediation mechanism between the branes. However, the action (1.4) does not tell the complete story, since it ignores the coupling to closed string modes that propagate in the bulk region between the branes.

In the following we will show how, via a simple topological arrangement, the bulk physics can enforce a low energy identification between $\mathcal{A}_{V}$ and $\mathcal{A}_{H}$. The basic mechanism is well known and makes use of the linear Chern-Simons-coupling of the gauge fields on the branes to the RR p-form field in the bulk. In a suitable geometrical set-up, this RR p-form field leads, upon $\mathrm{KK}$ reduction, to a massless 2 -form field $\mathcal{C}$ with a 4 -d action of the form ${ }^{1}$

$$
\mathcal{L}_{R R}=\mathcal{C} \wedge\left(d \mathcal{A}_{V}+d \mathcal{A}_{H}\right)+\frac{1}{2 \mu^{2}}|d \mathcal{C}|^{2} .
$$

This action can be recognized as a mass term for the gauge field $\mathcal{A}_{+}=\mathcal{A}_{V}+\mathcal{A}_{H}$. The simplest way to see this, is to rewrite $\Delta \mathcal{L}$ in the dual form

$$
\mathcal{L}_{R R}=\left(\mathcal{A}_{V}+\mathcal{A}_{H}\right) \wedge H+\frac{1}{2 \mu^{2}}|H|^{2}+d \varphi \wedge H
$$

where $H$ and $\varphi$ are independent fields. This action is gauge invariant, provided that the field $\varphi$ shifts under the U(1) gauge rotations. The equivalence of (1.6) to (1.5) is evident: the equation of motion of $\varphi$ reads $d H=0$, which can be solved by setting $H=d \mathcal{C}$. However, instead of solving for $H$, one may also integrate out $H$ and obtain

$$
\mathcal{L}_{R R}=\frac{1}{2} \mu^{2}\left|d \varphi+\mathcal{A}_{H}+\mathcal{A}_{V}\right|^{2}
$$

This is the well-known string mechanism for generating Stückelberg mass terms for abelian vector bosons. The mass scale $\mu$ is set by the size of the compactification manifold; for string scale size compactifications, $\mu$ is of order the string scale.

The upshot of the story is that, via the interaction between the branes and the RR-bulk fields, the linear sum $\mathcal{A}_{+}=\mathcal{A}_{V}+\mathcal{A}_{H}$ effectively decouples from the low energy physics, leaving a low energy action of the form (1.3) with

$$
\mathcal{A}^{\prime}=\mathcal{A}_{H}-\mathcal{A}_{V}
$$

Evidently, assuming that the hidden brane hosts a SUSY breaking sector, the system then provides a string realization of $\mathrm{U}(1)$ mediation. Somewhat unexpectedly, it arises as a bulk mediation mechanism. Pictorially, we can think of the mechanism as depicted in figure 1: the vector boson $\mathcal{A}_{1}$ on the visible brane can, via the linear CS-coupling, convert itself into an RR p-form that traverses the bulk towards the hidden brane. Here it can pick up a SUSY-breaking contribution, which via the same RR p-form mechanism, can be transported back to the visible sector. It is important to note that the mechanism only

\footnotetext{
${ }^{1}$ This interaction between abelian gauge bosons and 2-form fields is familiar as part of the Green-Schwarz anomaly cancelation mechanism 14] for anomalous U(1)'s, and results in a mass term for the corresponding vector boson [15, 16]. However, as is well known (and will be explained later), the RR-form mechanism for generating U(1) masses applies equally well to non-anomalous U(1)s 1, 17, 18]. We will focus on the case where the $\mathrm{U}(1)$ is non-anomalous.
} 


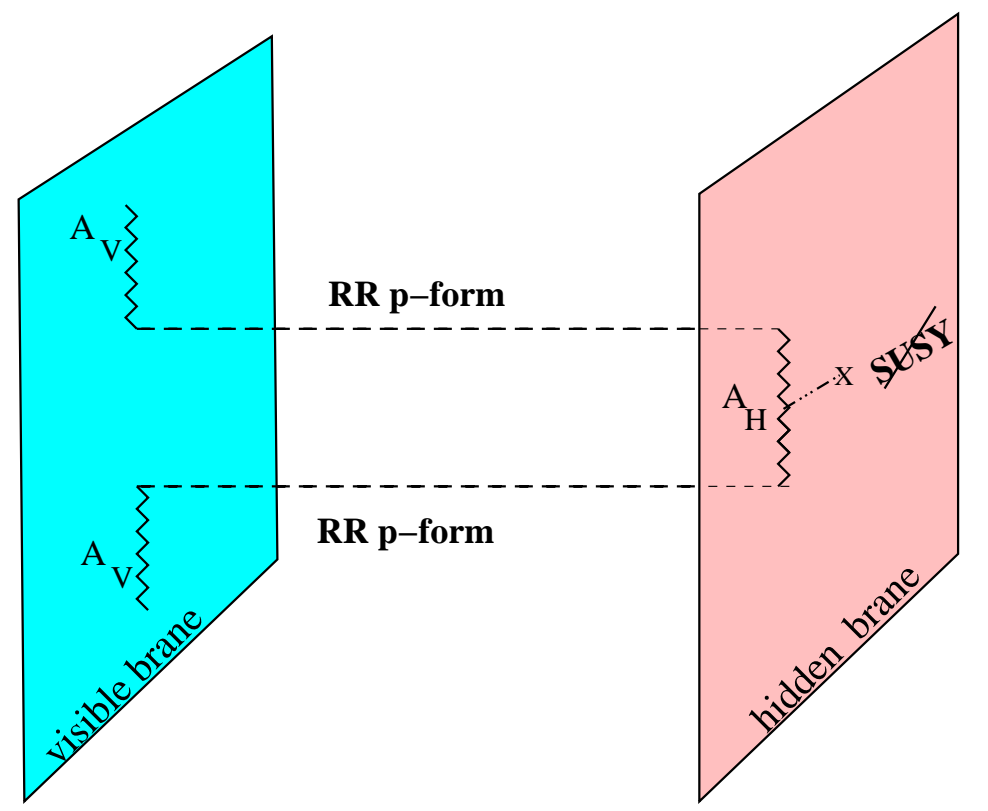

Figure 2: SUSY breaking on the hidden brane gets mediated to the visible sector via an RR p-form. It manifests itself via a mass-splitting between the abelian vector boson $\mathcal{A}_{1}$ and its superpartner.

works for abelian vector bosons, since the RR p-forms do not have a linear CS-coupling to non-abelian gauge bosons.

This paper is organized as follows. In section 2, after a short review the basic building blocks of string phenomenology with D-branes, we present some more details of the RRform mediation mechanism. In section 3 we discuss some possible applications of our result. In the appendix, we summarize how $\mathrm{U}(1)$ mediation can arise in the heterotic string.

\section{RR-form mediation}

In this section we will display the RR-form mediation mechanism in a specific IIB string theory set-up. Although the main ingredients and arguments are all quite familiar to experts, the story has sufficiently many instructive aspects to warrant a more detailed exposition.

Our set-up is as in figure 2: we imagine that the MSSM and the SUSY breaking hidden sector each live on separate (collections of) branes localized within separate small regions of the Calabi-Yau manifold. We will demonstrate how, in this generic set-up, the bulk physics may enforce a low energy identification between two abelian vector bosons $\mathcal{A}_{V}$ and $\mathcal{A}_{H}$ that live on the two separate branes. The key step will be to arrange things such that the gauge field that gets a mass is a linear sum of the two gauge fields. To set the stage, we first summarize some relevant ingredients of local brane constructions of 4-d gauge theories.

\subsection{Phenomenology with branes}

Engineering of 4-d gauge theories via D-branes has become a quite well-developed technology. Enabled by a growing assortment of available ingredients — brane intersections, 
branes at singularities, orientifolds - a rich landscape of gauge theories has been constructed, including an increasing number that closely resemble the MSSM [1-3]. While string phenomenology with D-brane does not readily encompass grand unified models or enforce gauge coupling unification, it has several other advantages. In particular, it is well suited for a bottom up strategy, that aims to construct the MSSM in terms of open string modes, that are hierarchically and geometrically separated from the string scale dynamics that determines the shape of the compactification manifold. Such a dynamical decoupling limit can be accomplished by localizing the brane configuration inside a small region of the Calabi-Yau, possibly at the bottom of a warped throat.

We will work in type IIB string theory, but a similar story holds for the IIA case. (We will comment on the IIA case later.) IIB string theory has Dp-branes with $p$ any odd integer from 1 to 9 . A typical IIB brane configuration will involve combination of D3, D5 and D7-branes, that fill our 3+1 space-time, while their remaining dimensions wrap internal compact cycles. Chiral matter arises from open strings that live at the intersection between D7 and D5 branes. $^{2}$ Due to the interaction with the local curved geometry, the Dbranes will in general form bound states, known as fractional branes 15$]$. Each bound state has D3, D5 and D7 brane components, that respectively wrap 0-, 2- or 4-cycles supported inside the local geometry, see figure $4 .^{3}$

The world-volume theory on a collection of fractional branes takes the general form of a quiver gauge theory [15]: each fractional brane gives rise to a $\mathrm{U}(N)$ gauge factor, with $N$ its multiplicity, and every intersection between two branes produces a chiral matter multiplet in the corresponding bi-fundamental representation. These rules can be generalized by including orientifold planes. The challenge of open string phenomenology is to find a suitable Calabi-Yau singularity and collection of fractional branes, such that the worldbrane theory takes the form of the MSSM, or some sufficiently realistic extension thereof [1].

Most D-brane constructions of MSSM-like theories involve extra U(1) factors besides the hypercharge symmetry. The extra $\mathrm{U}(1)$ gauge bosons typically acquire a string scale mass via coupling to closed string RR-form fields. (We will review this mechanism below.) Which U(1) factors actually survive as low energy gauge symmetries depends on the topology of how the local CY singularity is embedded inside of the full compact CY geometry [17, 18]. The common occurrence of extra U(1) factors in brane constructions motivates our present study of their possible role in the mediation of supersymmetry breaking.

\section{$2.2 \mathrm{U}(1)$ mediation via RR-forms}

Now we turn to the stringy $U(1)$ mediation mechanism. For concreteness, we assume that the visible $\mathrm{U}(1)$ vector boson $\mathcal{A}_{V}$ lives on a D5 brane, that wraps a 2-cycle $\mathrm{A}_{V}$ supported within the visible region $\varnothing$ of the compactification manifold (see figs 1 and 3 ). Similarly,

\footnotetext{
${ }^{2}$ Note that D5 and D7-branes have 2 and 4 internal dimensions, resp. Since $(2+4)$ equals the dimension of the internal CY manifold, their intersection looks like a point inside the CY but fills the 3+1-d space-time.

${ }^{3}$ The name fractional brane is chosen, because via the rearrangement of constituent branes into bound states combinations, a single D3-brane on a CY singularity may split into several fractional branes. Even a single D3 can thus support a rich spectrum of gauge and chiral matter. Examples of D3 brane theories with MSSM like matter have been considered in 3 .
} 


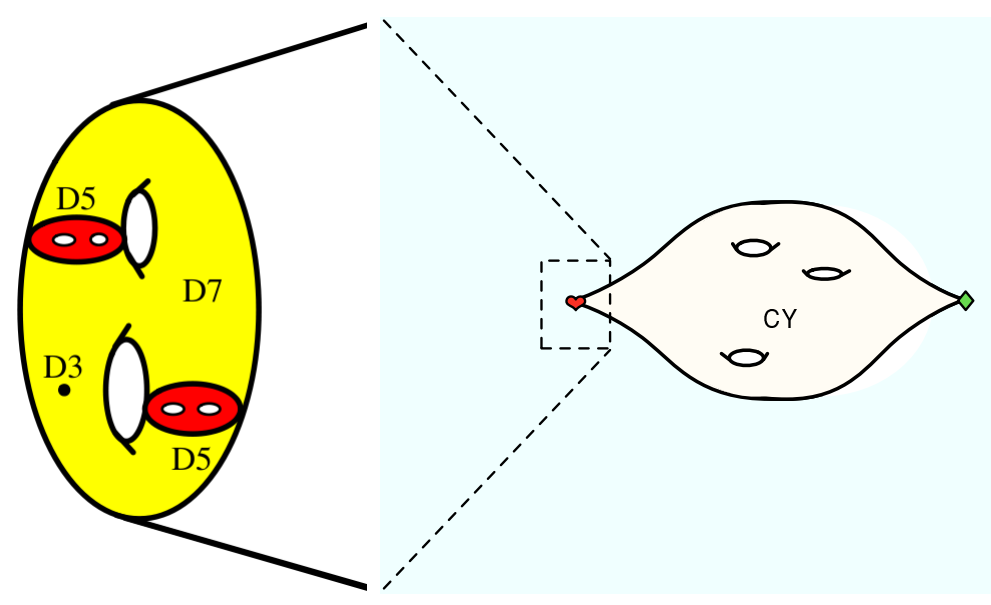

Figure 3: A typical localized IIB brane configuration consists of bound state combinations of D3, D5 and D7-branes wrapping 0-, 2- and 4-cycles, supported in a local region of the Calabi-Yau geometry. The local region may lie at the tip of a warped throat.

the $\mathrm{U}(1)$ vector boson $\mathcal{A}_{H}$ on the hidden brane formation is assumed to arise from open strings on a D5 brane, that wraps a 2-cycle $A_{H}$, supported within the local region $\diamond$. Note that the D5-branes are not placed in isolation, but are part of a bound state configuration of branes that hosts all other visible and hidden matter. The opens strings supported at the intersection of the D5 and D7-branes in the visible region represent chiral matter charged under $\mathcal{A}_{V}$.

For localized brane constructions, the Kähler area ${ }^{4}$ of the 2-cycles $\mathrm{A}_{i}$ represents the FI parameter on the the world volume gauge theory. So if this area is finite, it results in a non-zero FI-term ${ }^{5}$ that via the D-term constraint dictates non-zero expectation values for charged matter fields (produced by open string modes located at brane intersections). This breaks the $\mathrm{U}(1)$ gauge symmetry and renders the abelian gauge boson massive via a conventional Higgs mechanism. In the following, however, we will focus on another mechanism for giving a mass to the abelian vector bosons.

Consider a single isolated D5-brane wrapped on a 2-cycle A. (We will return to our actual set-up with a hidden and visible brane momentarily.) The field theory on the brane is described by a Born-Infeld action together with a Chern-Simons term. The CS term contains a linear coupling

$$
C_{4} \wedge d \mathcal{A}
$$

between the world-volume gauge field $\mathcal{A}$ and the RR 4 -form field $C_{4}$. The 5-form field strength of $C_{4}$ is self-dual in 10 dimensions

$$
F_{5}=* F_{5}, \quad F_{5}=d C_{4}
$$

\footnotetext{
${ }^{4}$ This area is defined as $\left|\int_{\mathbf{A}}(J+i B)\right|$ with $J$ the Kähler 2-form and $B$ the NS 2-form field.

${ }^{5}$ One intuitive way to quantify this correspondence is to consider a $D 3$-brane that wraps the 2-cycle A. In 3+1-dimensions, it reduces to a D-string with string tension proportional to the area of A, while in the gauge theory it represents a D-term string, with tension proportional to the FI-term.
} 
Although $C_{4}$ lives in the bulk, due to the linear CS coupling it can directly affect the spectrum of open string modes on the brane.

There is no covariant action that produces the self-duality equation (2.1) as its equation of motion 19]. However, we can think of the self-dual theory as the chiral half of the nonchiral theory defined by

$$
\mathcal{L}_{10-\mathrm{d}}=\frac{1}{2}\left|F_{5}\right|^{2}+d C_{4} \wedge F_{5}
$$

were the fields $F_{5}$ and $C_{4}$ (initially) need to be viewed as independent variables. The $C_{4}$ equation of motion dictates that $d F_{5}=0$, which is the Bianchi identity that tells us that $F_{5}$ is the exterior derivative of a 4 -form. The self-duality projection is implement by the constraint that $F_{5}=d C_{4}$.

To be consistent with self-duality, we need to slightly rewrite the CS interaction term as

$$
\mathcal{L}_{\mathrm{CS}}=C_{4} \wedge \mathcal{F}+\mathcal{A} \wedge F_{5}
$$

where $\mathcal{F}=d \mathcal{A}$ is the abelian field strength. The interaction term (2.3) adds source terms to the equations of motion for $C_{4}$ and $F_{5}$ (here $\delta_{\mathcal{M}}$ is the $\delta$-function localized on the D5 world-volume $\mathcal{M}$ )

$$
d F_{5}=\mathcal{F} \wedge \delta_{\mathcal{M}}, \quad * F_{5}-d C_{4}=\mathcal{A} \wedge \delta_{\mathcal{M}}
$$

These equations are clearly consistent with the self-duality constraint $F_{5}=* F_{5}$.

The field strength $F_{5}$ is gauge invariant under $\mathrm{U}(1)$ gauge transformations. However, we see that due to the explicit appearance of the abelian gauge potential $\mathcal{A}$, the interaction term (2.3) and resulting equations (2.4) are gauge invariant only under the combined gauge transformation

$$
\begin{aligned}
\mathcal{A} & \rightarrow \mathcal{A}+d \lambda, \quad d \wedge\left(d \eta+\lambda \delta_{\mathcal{M}}\right)=0 . \\
C_{4} & \rightarrow C_{4}+d \eta,
\end{aligned}
$$

This demonstrates that the $\mathrm{RR} 4$-form $C_{4}$ contains the longitudinal mode of $\mathcal{A}$, which is a first indication that the linear CS-coupling is capable of producing a mass term for $\mathcal{A}$.

To isolate the longitudinal mode, consider the 4-cycle B with the Calabi-Yau manifold that is dual to the 2-cycle A. The B-period of $C_{4}$ defines a 4 -d scalar

$$
\varphi=\int_{\mathrm{B}} C_{4}
$$

Using that B intersects the D5 world volume $\mathcal{M}$ at a single point, we deduce that $\varphi$ transforms under $\mathrm{U}(1)$ gauge rotations as

$$
\varphi \rightarrow \varphi-\lambda
$$

The 4-d action for $\varphi$ thus necessarily involves the gauge invariant combination $d \varphi+\mathcal{A}$.

Upon KK reduction, the RR 4-form field $C_{4}$ gives rise to a massless 2-form field for every 2-cycle within the CY manifold and a massless scalar mode for every 4-cycle. To simplify our formulas, let us assume that there is only one such dual pair, the 2-cycle A wrapped 
by the single D5 and its dual 4-cycle B. The associated massless 2-form field is obtained via

$$
\mathcal{C}=\int_{\mathrm{A}} C_{4}
$$

$\mathcal{C}$ is related to the scalar $\varphi$ in (2.6) via the 10 -d self-duality equation $F_{5}=* F_{5}$. To write this relation, we introduce the unique harmonic 2-form $\omega$ and 4 -form $\beta$ on the $\mathrm{CY}$, normalized such that

$$
\int_{\mathrm{A}} \omega=1, \quad \int_{\mathrm{B}} \beta=1, \quad \int_{C Y} \omega \wedge \beta=1 .
$$

The two forms are related via Hodge duality

$$
*_{6} \beta=\mu^{2} \omega, \quad \int_{\mathrm{CY}} \omega \wedge *_{6} \omega=\frac{1}{\mu^{2}} .
$$

Now, to perform the KK-reduction, we expand

$$
F_{5}=d \mathcal{C} \wedge \omega+(d \varphi+\mathcal{A}) \wedge \beta+\ldots
$$

We read off that $F_{5}$ is self-dual, provided we identify

$$
*_{4} d \mathcal{C}=\mu^{2}(d \varphi+\mathcal{A}) .
$$

Let us now return to our phenomenological set-up with a D5 wrapping a 2-cycle $A_{V}$ in the visible region and a D5 wrapping a 2-cycle $A_{H}$ in the hidden region. In case $A_{V}$ and $A_{H}$ were both distinct homology cycles within the CY, then after KK-reduction, the two world volume gauge fields $\mathcal{A}_{H}$ and $\mathcal{A}_{H}$ would couple to distinct 2-form modes $\mathcal{C}_{V}$ and $\mathcal{C}_{H}$. This is not our situation of interest. Instead, we will arrange things such that the KK reduction produces a single 2 -form mode $\mathcal{C}$, that couples to a linear combination of $\mathcal{A}_{V}$ and $\mathcal{A}_{H}$. This is naturally achieved as follows.

Although the hidden and visible regions may be located on opposite sides of the CY manifold, it is possible to choose the CY geometry such that the 2-cycles $A_{V}$ and $A_{H}$ are in fact topologically the same 2-cycle, so that, as elements in $H_{2}(C Y)$,

$$
A_{V}=A_{H} .
$$

This equation states that a D5 wrapping $A_{V}$ can be continuously deformed into a D5 wrapping $A_{H}$. During this deformation, the D5 would first need to expand while moving away from the visible region, then traverse the CY and finally contract to wrap a small 2-cycle near the hidden region. So each brane locally minimizes its energy. This situation is schematically depicted in fig 4.

The two homologous 2-cycles $A_{V}$ and $A_{H}$ share the same dual harmonic 2-form $\omega$, and the same dual 4-cycle $B$ and associated harmonic 4 -form $\beta$. We again make the simplifying assumption that these are the only non-trivial 2- and 4-cycles around. The KK reduction of the 4 -form $C_{4}$ then proceeds as outlined above, except that we must include the CScoupling to both the visible and hidden branes. As a result, the 4-d duality relation (2.12) gets modified to

$$
*_{4} d \mathcal{C}=\mu^{2}\left(d \varphi+\mathcal{A}_{V}+\mathcal{A}_{H}\right) .
$$




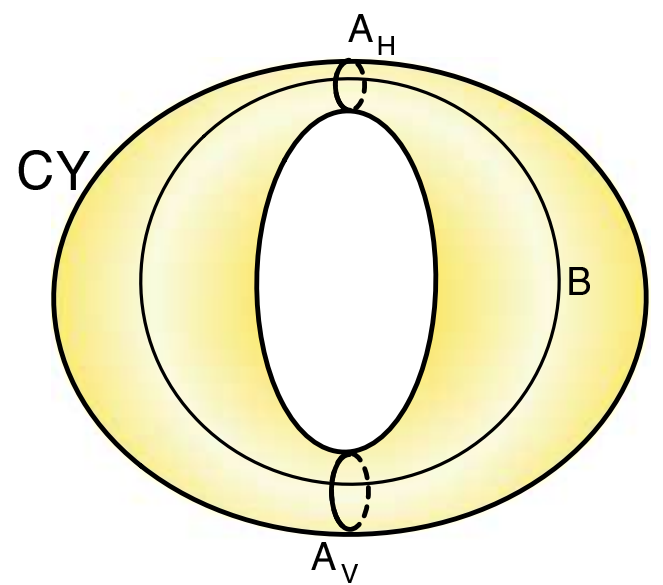

Figure 4: A schematic depiction of our topological set-up. The visible and hidden D-branes wrap homologous cycles $A_{V}$ and $A_{H}$ within the $C Y$ manifold, that share a common dual cycle $B$.

The rest of the story now proceeds as summarized in the introduction: the KK reduction yields a 4 -d action for the 2 -form field $\mathcal{C}$ of the form (1.5), where $\mu^{2}$ is defined in (2.10). The above relation (2.14) arises as the solution to equation of motion of the dual action (1.6). Eliminating $H$ from the action (1.6) results in the Stückelberg mass term for $\mathcal{A}_{V}+\mathcal{A}_{H}$.

More insight is obtained by considering the superfield version of the story [15]. The field $\varphi$ arises as the imaginary part of a complex superfield $T$, one of the Kähler moduli that parametrizes the size of the compact CY manifold. The real bosonic part of $T$ equals the volume (in the Einstein frame) of the 4-cycle B

$$
T=\phi+i \varphi=\int_{\mathrm{B}}\left(\sqrt{g}+i C_{4}\right) .
$$

The 4-d action of $T$ is obtained via KK reduction of the IIB supergravity action. Supersymmetry and gauge invariance dictate that its kinetic term takes the generic form

$$
\int d^{4} \theta K\left(T+\bar{T}+V_{V}+V_{H}\right)
$$

where $V_{V}$ and $V_{H}$ denote the vector multiplets on the visible and hidden D5-brane. Upon performing the superfield integral, $K$ yields for the bosonic kinetic term

$$
K^{\prime \prime}(\phi)\left(\left|d \varphi+\mathcal{A}_{V}+\mathcal{A}_{H}\right|^{2}+|d \phi|^{2}\right)+K^{\prime}(\phi)\left(\mathcal{D}_{V}+\mathcal{D}_{H}\right) .
$$

This includes a mass term for $\mathcal{A}_{+}=\mathcal{A}_{V}+\mathcal{A}_{H}$. This mass in essence arises via a conventional Higgs mechanism, with $\Phi=e^{-T}$ playing the role of the charged Higgs superfield. ${ }^{6}$ In a complete setting, the real modulus field $\phi$ is - via a modulus stabilzation mechanism driven by the coupling to other string scale degrees of freedom - stabilized at some non-vanishing expectation value $\langle\phi\rangle$. This translates into a finite vacuum condensate for the charged Higgs

\footnotetext{
${ }^{6}$ The complex superfield $T$ is equal to the action of a euclidean D3-brane instanton that wraps the 4-cycle B; contributions to the effective action involving the Higgs field $\Phi=e^{-T}$ can be viewed as the contributions due to D3-brane instantons.
} 
superfield $\Phi$, that spontaneously breaks the U(1) gauge symmetry and produces the mass term for $\mathcal{A}_{+}$

Eq. (2.16) indicates that the coupling to the modulus $\langle\phi\rangle$ may also induce an FI-term for the hidden and visible brane theory. If there is $\mathrm{U}(1)$ charged chiral matter present on either the visible or hidden brane, the D-term constraint then enforces that some of these charged scalar fields must develop a vacuum expectation value. This is another mechanism for giving mass to the $\mathrm{U}(1)$ bosons, distinct from the bulk mechanism outlined above. For $\mathrm{U}(1)$ mediation, we need to assume that these D-term driven masses are small compared to the SUSY breaking scale, possibly by making certain choices of the content of the hidden sector.

\subsection{Low energy parameters}

In this section we will discuss how SUSY breaking may get mediated from the hidden to the visible brane, from the low energy point of view. Our discussion will be brief, because after decoupling the heavy $\mathrm{U}(1)$ gauge boson $\mathcal{A}_{+}$, the mechanism simply reduces to $\mathrm{U}(1)$ mediation via the light gauge boson $\mathcal{A}_{-}$.

The gauge couplings of D-brane gauge fields are governed by closed string moduli that paramerize the size of the cycles wrapped by the D-branes, and by the periods of the NS 2 form $B$. The kinetic term of the two U(1) gauge fields take the form

$$
\mathcal{W}=\sum_{i=H, V} \frac{f_{i}\left(S_{i}, X_{i}\right)}{4} W_{i}^{\alpha} W_{i, \alpha}+\text { h.c. }
$$

where $S_{i}, i=V, H$ are closed string moduli fields, and $X_{i} i=V, H$ other open string matter fields, that may contribute to supersymmetry breaking and mediation. The gauge-coupling is given by The moduli $S_{i}$ are stabilized at some non-zero expectation value. The U(1) gauge couplings are then given by

$$
g_{i}^{2}=\frac{1}{\operatorname{Re} f_{i}\left(\left\langle S_{i}\right\rangle\right)}, \quad i=V, H .
$$

Hence, after canonically normalizing the gauge-fields, the heavy linear combination reads

$$
\begin{aligned}
& \mathcal{A}_{+}=\frac{1}{\sqrt{g_{V}^{2}+g_{H}^{2}}}\left(g_{V} \mathcal{A}_{V}+g_{H} \mathcal{A}_{H}\right) . \\
& \mathcal{A}^{\prime}=\frac{1}{\sqrt{g_{V}^{2}+g_{H}^{2}}}\left(g_{H} \mathcal{A}_{V}-g_{V} \mathcal{A}_{H}\right) .
\end{aligned}
$$

The heavy field $\mathcal{A}_{+}$has a mass $m_{+}=\mu \sqrt{g_{V}^{2}+g_{H}^{2}}$ with $\mu$ defined in eq. (2.10). The expression for $\mu$ in terms of geometric shape of the Calabi-Yau geometry depends on details of the supergravity reduction, that we will not attempt to evaluate here. It is clear, however, that for string size compactifications, the mass is of order the string scale. ${ }^{7}$

\footnotetext{
${ }^{7}$ This conclusion is not dependent on whether the D-branes are localized at the bottom of a warped throat. The reason is that the integral (2.10) is not a localized 5-d mass term.
} 
The $\mathcal{A}_{+}$gaugino marries off to the fermionic component of $T$ to form a Dirac fermion with a mass of the same magnitude. We will therefore assume that the heavy vector mutliplet decouples from the low energy physics. ${ }^{8}$ The low energy spectrum thus only contains the other linear combination $\mathcal{A}_{-}$and the corresponding light gaugino.

The $\mathrm{U}(1)$ gaugino can receive a mass via gravity mediation or gauge mediation, or a combination of the two. In both cases, we imagine that the SUSY breaking contributions originate in the hidden region, and first communicate their effect to the hidden sector brane. Let $F_{S}$ denote the F-term contribution to the hidden closed string modulus $S_{H}$, and $F_{X}$ the F-term of the hidden matter field $X_{H}$, generated via possible gauge mediated contributions to supersymmetry breaking. Then the $\mathrm{U}(1)$ gaugino mass is (up to corrections suppressed by the heavy linear combination's mass),

$$
M_{\lambda^{\prime}}=\frac{g_{V}^{2}}{g_{V}^{2}+g_{H}^{2}}\left(F_{S} \partial_{S} f_{H}+F_{X} \partial_{X} f_{H}\right)
$$

Summarizing, the parameters relevant for the low energy theory are the gauge-coupling of the light linear combination,

$$
g^{\prime}=\frac{g_{H} g_{V}}{\sqrt{g_{H}^{2}+g_{V}^{2}}},
$$

and the corresponding gaugino mass eq. (2.21). In addition, there can be a D-term, as read off from eq. (2.16). If the D-term is small compared with the gaugino soft mass then one is lead to the model recently considered in [11. If more structure is added then models such as the one considered in [21] can be constructed.

In the set-up as discussed so far, we have assumed that the hidden and visible gauge bosons are associated with non-anomalous gauge symmetries. In principle, it is also possible to consider the case when the hidden and visible $\mathrm{U}(1)$ are both anomalous. The RR-form mechanism for generating a U(1) masses is indeed a familiar part of the Green-Schwarz anomaly cancelation mechanism. Models of SUSY breaking and mediation with anomalous $\mathrm{U}(1)$ 's has been previously studied in [22, 23].

\section{Summary and discussion}

We have shown that string phenomenological scenarios with visible and hidden sector localized on D-branes, the coupling of the D-brane gauge theory to RR p-forms in the bulk gives rise to a robust SUSY breaking mediation mechanism, that remains effective when the two sectors are separated by a large distance compared to the string length. Our exposition specialized to U(1) gauge theories on D5-branes in IIB string theory, but the same mechanism works in type IIA and heterotic string theory. In IIA constructions, the gauge theory typically lives on D6-branes, which have a linear CS coupling $C_{5} \wedge F$ to an RR 5-form. In case the visible and hidden sector D6-branes wrap homologous 3-cycles, the RR coupling produces a mass-term for the diagonal combination of the two vector

\footnotetext{
${ }^{8}$ In scenarios with low string scale, the heavy field may still be relevant, as discussed in [16].
} 
bosons, leading to a low energy action of the form (1.3) suitable for U(1) mediation. In the appendix, we outline how $\mathrm{U}(1)$ mediation is naturally realized in the heterotic string.

We have not been specific about how supersymmetry may be broken on the hidden brane. There are several possibiliities one could contemplate. Which of these is most adequate may depend on the required scale of SUSY breaking, and on whether U(1) mediation acts in combination with other mechanisms or is the main mechanism responsible for generating the soft parameters.

In pure U(1) mediation, one only needs a relatively low SUSY breaking scale, but one needs the ability to tune this scale to a high accuracy [11]. As a possible realization, we can look for a gauge theory on the hidden sector brane that spontaneously breaks SUSY. The D-brane technology for engineering such gauge theories has substantially improved over the last year, and a growing number of examples have been found [24]. We can now imagine placing one of these SUSY breaking D-brane systems at the tip of a warped deformed conifold [12, 13]. In this way, one can discretely tune the SUSY breaking scale in the hidden sector by appropriately adjusting the quantized 3-form fluxes that support the conifold.

Alternatively, one can consider scenarios that combines U(1) mediation with another mechanism, such as gauge mediation or anomaly mediation. Like gauge mediation, U(1) mediation works for relatively low SUSY breaking scales. Moreover, in D-brane models of gauge mediation, the visible and hidden sector are living on nearby stacks of branes, one may easily encounter situations where both sectors have a common $U(1)$ factor.

$\mathrm{U}(1)$ mediation may also mix with anomaly mediation. The two form a natural pair, since both are flavor blind bulk mediation mechanisms that can communicate SUSY breaking between geometrically sequestered sectors. It was recently shown that the sequestering, that is a necessary precondition for anomaly meditation, can be realized in string compactifications where the SUSY breaking takes place at the bottom of a warped conifold. In case a visible U(1) gauge coupling gets a contribution from a hidden sector located in the nonSUSY region, one can imagine that sequestering fails for this particular coupling only. The spectrum and phenomenology of this mixed scenario, for the special case of hypercharge $\mathrm{U}(1)_{Y}$ mediation, is studied in 25].

\section{Acknowledgments}

We acknowledge useful discussions with R. Dermisek, P. Langacker, G. Paz, and C. Vafa. This work was supported by the National Science Foundation under grant PHY-0243680. Any opinions, findings, and conclusions or recommendations expressed in this material are those of the authors and do not necessarily reflect the views of the National Science Foundation.

\section{A. $U(1)$ mediation in the heterotic string}

Although our focus in this paper is on the type II setting with D-branes, the mechanism we have discussed can also be implemented in the heterotic string. 
Consider the $E_{8} \times E_{8}$ heterotic string compactified on a Calabi-Yau manifold. In order to get chiral matter we turn on holonomy for the ten-dimensional gauge groups and solve the Dirac equation for the ten-dimensional gaugino in this background. The low energy gauge group is the commutant of the holonomy group in $E_{8} \times E_{8}$. This gives rise to two four-dimensional sectors, one sector descending from the first $E_{8}$ which will be our visible sector, and the other from the second $E_{8}$ which will be the hidden sector where SUSY breaking takes place.

If the holonomy group includes a $\mathrm{U}(1)$ factor, i.e. there is a non-trivial line bundle $L$ with first Chern class $c_{1}(L)$, then this $\mathrm{U}(1)$ is also part of the unbroken gauge group since it commutes with the holonomy. However the 10-d action has couplings of the form

$$
\mathcal{L}_{10} \supset\left(d B-\omega_{L}-\omega_{R}\right)^{2}
$$

where $\omega_{L, R}$ are the Chern-Simons three-forms for the two $E_{8}$ gauge groups. Upon KK reduction of the $\mathrm{B}$-field along the harmonic form in the class $c_{1}(L)$ we get a non-universal axion with a Stückelberg coupling to our U(1) gauge field. Thus the unbroken U(1) generically gets a large mass and is removed from the low energy spectrum.

However, analogous to the type II set-up, we may also turn on a line bundle $L^{\prime}$ in the hidden $E_{8}$ with first Chern class $\left[c_{1}(L)\right]=\left[c_{1}\left(L^{\prime}\right)\right]$, which yields a corresponding unbroken $\mathrm{U}(1)$ in the hidden sector. The ten-dimensional couplings (A.1) give rise to

$$
\mathcal{L}_{4} \quad \supset\left(\partial_{\mu} a-A_{\mu}^{L}-A_{\mu}^{R}\right)^{2}
$$

in four dimensions. Thus we see that the linear combination $A_{\mu}^{L}+A_{\mu}^{R}$ swallows the axion $a$ and gets a large mass, and we are left with the linear combination $A_{\mu}^{L}-A_{\mu}^{R}$ which couples to charged matter both in the hidden and the visible sector. We can still give this extra $Z^{\prime}$ a mass by a conventional Higgsing in the hidden sector.

This method for generating massless U(1)'s has been known since the early days of heterotic model building (see eg. [26], where the role of the massless U(1) was played by hypercharge). However it does not seem to have been considered in the context of mediation of SUSY breaking. For more recent work on heterotic model building with U(1) factors, see [27].

\section{References}

[1] For recent reviews of string phenomenology with D-branes, see: R. Blumenhagen, B. Körs, D. Lüst and S. Stieberger, Four-dimensional string compactifications with D-branes, orientifolds and fluxes, Phys. Rept. 445 (2007) 1 hep-th/0610327;

R. Blumenhagen, M. Cvetič, P. Langacker and G. Shiu, Toward realistic intersecting D-brane models, Ann. Rev. Nucl. Part. Sci. 55 (2005) 71 hep-th/0502005.

[2] G. Aldazabal, L.E. Ibáñez, F. Quevedo and A.M. Uranga, D-branes at singularities: a bottom-up approach to the string embedding of the standard model, JHEP 08 (2000) 002 hep-th/0005067;

G. Aldazabal, L.E. Ibáñez and F. Quevedo, A D-brane alternative to the MSSM, JHEP 02 (2000) 015 hep-ph/0001083; 
G. Aldazabal, S. Franco, L.E. Ibáñez, R. Rabadán and A.M. Uranga, $D=4$ chiral string compactifications from intersecting branes, J. Math. Phys. 42 (2001) 3103 hep-th/0011073; L.E. Ibáñez, F. Marchesano and R. Rabadán, Getting just the standard model at intersecting branes, JHEP 11 (2001) 002 hep-th/0105155;

R. Blumenhagen, B. Körs, D. Lüst and T. Ott, The standard model from stable intersecting brane world orbifolds, Nucl. Phys. B 616 (2001) 3 hep-th/0107138;

M. Cvetič, G. Shiu and A.M. Uranga, Three-family supersymmetric standard like models from intersecting brane worlds, Phys. Rev. Lett. 87 (2001) 201801 hep-th/0107143; Chiral

four-dimensional $N=1$ supersymmetric type IIA orientifolds from intersecting D6-branes, Nucl. Phys. B 615 (2001) 3 hep-th/0107166.

[3] D. Berenstein, V. Jejjala and R.G. Leigh, The standard model on a D-brane, Phys. Rev. Lett. 88 (2002) 071602 hep-ph/0105042;

H. Verlinde and M. Wijnholt, Building the standard model on a D3-brane, JHEP 01 (2007) 106 hep-th/0508089.

[4] J.F.G. Cascales, M.P. Garcia del Moral, F. Quevedo and A.M. Uranga, Realistic D-brane models on warped throats: fluxes, hierarchies and moduli stabilization, JHEP 02 (2004) 031 hep-th/0312051.

[5] A. Anisimov, M. Dine, M. Graesser and S.D. Thomas, Brane world SUSY breaking from string/M theory, JHEP 03 (2002) 036 hep-th/0201256.

[6] K. Choi, A. Falkowski, H.P. Nilles and M. Olechowski, Soft supersymmetry breaking in KKLT flux compactification, Nucl. Phys. B 718 (2005) 113 hep-th/0503216.

[7] D.E. Diaconescu, B. Florea, S. Kachru and P. Svrček, Gauge - mediated supersymmetry breaking in string compactifications, JHEP 02 (2006) 020 hep-th/0512170].

[8] S. Kachru, L. McAllister and R. Sundrum, Sequestering in string theory, JHEP 10 (2007) 013 hep-th/0703105.

[9] L. Randall and R. Sundrum, Out of this world supersymmetry breaking, Nucl. Phys. B 557 (1999) 79 hep-th/9810155;

G.F. Giudice, M.A. Luty, H. Murayama and R. Rattazzi, Gaugino mass without singlets, JHEP 12 (1998) 027 hep-ph/9810442.

[10] M. Dine and A.E. Nelson, Dynamical supersymmetry breaking at low-energies, Phys. Rev. D 48 (1993) 1277 hep-ph/9303230;

M. Dine, A.E. Nelson and Y. Shirman, Low-energy dynamical supersymmetry breaking simplified, Phys. Rev. D 51 (1995) 1362 hep-ph/9408384;

M. Dine, A.E. Nelson, Y. Nir and Y. Shirman, New tools for low-energy dynamical supersymmetry breaking, Phys. Rev. D 53 (1996) 2658 hep-ph/9507378;

For a review of gauge mediation, see: G.F. Giudice and R. Rattazzi, Theories with gauge-mediated supersymmetry breaking, Phys. Rept. 322 (1999) 419 hep-ph/9801271.

[11] P.G. Langacker, G. Paz, L.-T. Wang and I. Yavin, Z'-mediated supersymmetry breaking, Phys. Rev. Lett. 100 (2008) 041802 arXiv:0710.1632].

[12] I.R. Klebanov and M.J. Strassler, Supergravity and a confining gauge theory: duality cascades and $\chi S B$-resolution of naked singularities, JHEP 08 (2000) 052 hep-th/0007191.

[13] S.B. Giddings, S. Kachru and J. Polchinski, Hierarchies from fluxes in string compactifications, Phys. Rev. D 66 (2002) 106006 hep-th/0105097. 
[14] M.B. Green and J.H. Schwarz, Anomaly cancellation in supersymmetric D $=10$ gauge theory and superstring theory, Phys. Lett. B 149 (1984) 117.

[15] M.R. Douglas and G.W. Moore, D-branes, quivers and ALE instantons, hep-th/9603167.

[16] D.M. Ghilencea, L.E. Ibáñez, N. Irges and F. Quevedo, TeV-scale Z' bosons from D-branes, JHEP 08 (2002) 016 hep-ph/0205083.

[17] H. Jockers and J. Louis, D-terms and F-terms from D7-brane fluxes, Nucl. Phys. B 718 (2005) 203 hep-th/0502059; The effective action of D7-branes in $N=1$ Calabi-Yau orientifolds, Nucl. Phys. B 705 (2005) 167 hep-th/0409098].

[18] M. Buican, D. Malyshev, D.R. Morrison, H. Verlinde and M. Wijnholt, D-branes at singularities, compactification and hypercharge, JHEP 01 (2007) 107 hep-th/0610007.

[19] N. Marcus and J.H. Schwarz, Field theories that have no manifestly Lorentz invariant formulation, Phys. Lett. B 115 (1982) 111.

[20] Z. Chacko, M.A. Luty and E. Ponton, Massive higher-dimensional gauge fields as messengers of supersymmetry breaking, JHEP 07 (2000) 036 hep-ph/9909248].

[21] D.E. Kaplan, F. Lepeintre, A. Masiero, A.E. Nelson and A. Riotto, Fermion masses and gauge mediated supersymmetry breaking from a single U(1), Phys. Rev. D 60 (1999) 055003 hep-ph/9806430.

[22] P. Binetruy and E. Dudas, Gaugino condensation and the anomalous U(1), Phys. Lett. B 389 (1996) 503 hep-th/9607172.

[23] G.R. Dvali and A. Pomarol, Anomalous U(1) as a mediator of supersymmetry breaking, Phys. Rev. Lett. 77 (1996) 3728 hep-ph/9607383.

[24] Some recent papers on D-brane realization of gauge theories with broken SUSY are: M. Aganagic, C. Beem and S. Kachru, Geometric transitions and dynamical SUSY breaking, arXiv:0709.4277;

M. Buican, D. Malyshev and H. Verlinde, On the geometry of metastable supersymmetry breaking, arXiv:0710.5519.

[25] R. Dermisek, H. Verlinde and L.-T. Wang, Hypercharged anomaly mediation, arXiv:0711.3211.

[26] E. Witten, New issues in manifolds of SU(3) holonomy, Nucl. Phys. B 268 (1986) 79.

[27] R. Blumenhagen, G. Honecker and T. Weigand, Loop-corrected compactifications of the heterotic string with line bundles, JHEP 06 (2005) 020 hep-th/0504232];

R. Blumenhagen, S. Moster and T. Weigand, Heterotic GUT and standard model vacua from simply connected Calabi-Yau manifolds, Nucl. Phys. B 751 (2006) 186 hep-th/0603015]. 